EPJ Web of Conferences 59, 04004 (2013)

DOI: $10.1051 /$ epjconf/20135904004

(C) Owned by the authors, published by EDP Sciences, 2013

\title{
Analysis of mix experiments on Omega
}

\author{
P.A. Bradley a , J.A. Cobble, J.R. Fincke, S.C. Hsu, G.R. Maglessen, \\ T.J. Murphy, M.J. Schmitt, I.L. Tregillis, N.S. Vinyard, F.J. Wysocki \\ and K.D. Obrey
}

Los Alamos National Laboratory, MS F699, Los Alamos, NM 87545, USA

\begin{abstract}
A 2-D Eulerian code with a turbulent mix model was used for the first time to model a set of plastic $(\mathrm{CH})$ ablator capsules with $15 \mu \mathrm{m}$ thick $\mathrm{CH}$ shells. Our simulations of these capsules do a reasonable job of matching the implosion radius versus time, self-emitting core radius, and have an experiment/simulation yield ratio that is about 0.24 .
\end{abstract}

\section{INTRODUCTION}

Mix is a ubiquitous feature in ICF capsule implosions and degrades the neutron yield from the ideal "clean calculation" limit. Generally, mix becomes more dominant with increasing convergence ratio $[1,2]$. Until recently, simulations did not include the effects of mix and even now, the mix prescriptions are either approximate or are applicable only in one dimension (1-D) [4-7]. We have not seen any reference to 2-D calculations with a subgrid turbulent mix model in the literature, although Hammel et al. [6] uses a large eddy model in their simulations. The simulations described in this paper are 2-D and use the BHR-2 turbulent mix model [8].

The Defect Induced Mix Experiment (DIME) shots on the Omega laser [9] were designed to validate our mix models so that we can understand mix in convergent geometries and ultimately feature-driven mix. In this paper, we describe Omega experiments involving spherical capsules without defects that are a first step in our experimental plan.

\section{THE EXPERIMENTS}

We discuss four spherical capsules with a $\mathrm{CH}$ shell thickness of $\sim 15 \mu \mathrm{m}$ fielded in April 2009 and September 2010. These capsules were shot at the Omega laser in the symmetric 60 beam direct drive configuration. The laser pulse was a $1 \mathrm{~ns}$ square shape with a nominal energy of $23 \mathrm{~kJ}$. Capsules like these typically absorb about 60 to $65 \%$ of the laser drive energy [11]. The targets were mounted on a $\mathrm{SiC}$ stalk that had a diameter of 17 to $20 \mu \mathrm{m}$ and was glued to the capsule with epoxy. The stalk and epoxy spot are three-dimensional and not modeled in our simulations.

We obtained implosion trajectories, neutron yields, burn-average ion temperatures, time of peak burn (also called bang time), and burn widths. We know the initial DT fill pressures (5 atm), capsule diameters, and shell thickness for all of the shots. Some of these quantities are shown in Table 1. The capsules were filled with DT gas a few weeks before the shots. We obtained an X-ray image for one capsule and we have radius versus time data for another capsule. One significant issue with the

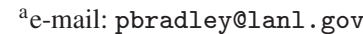

This is an Open Access article distributed under the terms of the Creative Commons Attribution License 2.0, which permits unrestricted use, distribution, and reproduction in any medium, provided the original work is properly cited. 
Table 1. Data from Omega shots.

\begin{tabular}{|l|c|c|c|c|c|c|}
\hline $\begin{array}{l}\text { Shot } \\
\text { number }\end{array}$ & Thickness & Diameter & Expt Yield & $\begin{array}{c}\text { Sim } \\
\text { Yield }\end{array}$ & Expt BangTime & Expt BurnWidth \\
\hline & $(\mu \mathrm{m})$ & $(\mu \mathrm{m})$ & neutrons & neutrons & $(\mathrm{ns})$ & $(\mathrm{ps})$ \\
\hline 54145 & 15.1 & 885 & $1.27 \mathrm{e} 13$ & $4.58 \mathrm{e} 13$ & 1.447 & 170 \\
\hline 54135 & 15.2 & 891 & $1.37 \mathrm{e} 13$ & $5.53 \mathrm{e} 13$ & 1.504 & 159 \\
\hline 59514 & 14.7 & 874 & $7.45 \mathrm{e} 12$ & $3.38 \mathrm{e} 13$ & 1.571 & 175 \\
\hline 59520 & 15.0 & 884 & $6.56 \mathrm{e} 12$ & $3.66 \mathrm{e} 13$ & 1.573 & 161 \\
\hline
\end{tabular}

September data is the bang times were consistently 100 to $150 \mathrm{ps}$ later and the yields were a factor of two lower than that of the nominally identical capsules fired in April 2009. We examined the capsule metrology, the laser drives, and capsule gas fills. The laser drive in September was $95 \%$ of the drive in April, but simulations show this produces only a $30 \%$ yield drop and 30 ps delay in bang time. The capsule dimensions and gas fill pressures are the same between April and September and are not responsible for the yield difference. The capsules fired in September 2010 were stored at -80C for about a year, and something about this storage caused the capsules to behave differently, but there is not enough information to determine the exact cause [12]. However, we are able to match the delayed bang times by reducing the energy source by $25 \%$. The reduced drive also reduces the calculated yield by about half, in rough agreement with the data. We are able to match the radius versus time data with the reduced drive simulation, giving us confidence that we are modeling the implosion correctly, even if we do not know the exact cause of the bang time delay and yield drop.

\section{SIMULATIONS AND COMPARISON TO DATA}

We used an adaptive mesh refinement (AMR) Eulerian code to simulate the experiments. This code can run the implosions through burn, but it lacks laser beams and laser energy deposition physics. Because of this, we use an energy source in a $7 \mu \mathrm{m}$ layer on the outside of the capsule, which gives the correct amount of energy absorbed (typically about 60 to $65 \%$ of the total laser energy [11]) and correct bang time. The source is spherically symmetric and does not include any beam imprinting or illumination irregularities (typically $\sim 2 \%$ ). The initial 2-D mesh consists of two capsule shell layers, one for the aforementioned source region $(7 \mu \mathrm{m})$ and the second layer that is the rest of the $\mathrm{CH}$ shell. There is also a central DT gas material and an outer low-density "vacuum" region for the ablated shell to expand into. The shell and gas regions have initial zones as small as $0.25 \mu \mathrm{m}$, although the cells can be up to $1 \mu \mathrm{m}$ wide in the middle of the shell and gas. 3-D features, such as the mounting stalk, are not included. We also do not include the $\sim 100 \mathrm{~nm}$ aluminum overcoat layer, as calculations show this has a negligible effect on the simulated implosion.

A common feature of ICF capsule simulations is that the observed yields are lower than the calculated yields, especially when mix is not included. Simulations without mix predict a yield that is about 20 times the observed yield. The turbulent mix model (called BHR-2 [8]) in our code has three initial conditions. The first is the roughness scale of the capsule surface, which we set to $25 \mathrm{~nm}$. This is the amplitude of the $1=10$ to 60 modes on the capsule. Simulations with the roughness set to 10 or $50 \mathrm{~nm}$ cause a $+/-14 \%$ change in yield. Another initial condition is the initial turbulent specific kinetic energy, which is set to $0.1 \%$ of the total kinetic energy based on turbulent fluid flow experiments [8]. Finally, we turn on the mix model when the inward shock is about to break through the shell, which occurs at $0.3 \mathrm{~ns}$.

We start by comparing the radius versus time information from the outer emitting corona of shot 59514 (see Fig. 1). The average difference between the simulation and experimental radius points is about $22 \mu \mathrm{m}$ (the measurement error of each point is about 5 microns). There are two core radius points 
IFSA 2011

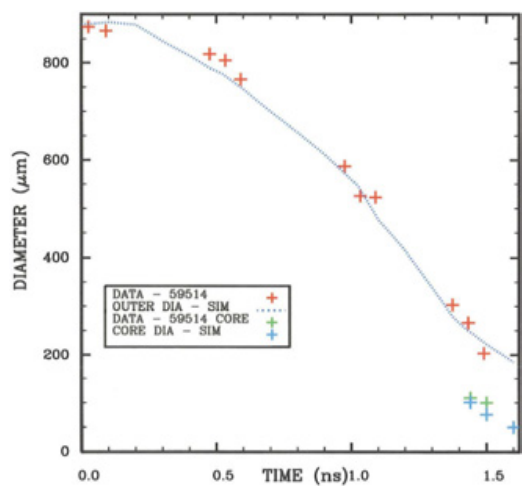

Figure 1. Comparison of emission $x$-ray radius versus time against a 2-D simulation. The average difference between the simulation (blue line) and experimental radius points (red "+") is about $22 \mu \mathrm{m}$. We see similar differences between experimental (green "+") and simulation (blue "+") self-emission core data points.

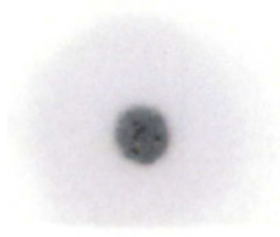

Figure 2. A comparison of self-emission x-ray image from a perfect capsule implosion at $1.55 \mathrm{~ns}$ (left panel) with a simulated image (right panel). The core radius of the data is $52 \mu \mathrm{m}$ while the simulation core has a radius of $30 \mu \mathrm{m}$.

(green "+" symbols); they are about $20 \mu \mathrm{m}$ larger than the simulation point (blue "+" symbols) on average. Finally, we have a self-emission x-ray image of shot 59520 at $1.55 \mathrm{~ns}(20$ ps before bang time). The experimental image has a radius of $51.6 \mu \mathrm{m}$. Our simulated $\mathrm{x}$-ray image (convolved with the same $60 \mathrm{ps}$ gate time as the data) has a radius of $30 \mu \mathrm{m}$. This result is consistent with the core radius differences in Fig. 1. Given our perfectly symmetric drive, we expect our simulations to converge too much (assuming no preheat of the shell). Simulations of other capsules without perfectly symmetric drive produce images that are only about 20\% smaller that the data. The simulated image in Fig. 2 also has a pronounced octagonal shape, which is an artifact of the Cartesian mesh distorting the flow on the 0,45 , and $90^{\circ}$ positions.

We continue our comparison with the yields (see Table 1). The ratio of the experimental yield to the mix simulation yield is 0.20 to 0.28 for all four shots. The September 2010 shots have experimental yields that are 53\% that of the April 2009 capsules, whereas our calculated yields for the September 2010 shots are $70 \%$ of the April 2009 yields. The simulation yield drop is due to the weaker shock launched from a weaker source, which also delays the simulated bang time. The simulation $T_{\text {ion }}$ values are $2.5 \mathrm{keV}$ higher than the data on average and the calculated burn widths are 59 to $64 \%$ as wide as the data. The high $\mathrm{T}_{\mathrm{ion}}$ value and narrow burn width are consistent with a calculation that converges too much, since the extra convergence implies too high a density, which would increase the burn rate and the yield. If we assume that the burn rate (and the yield) are proportional to the square of the density, then the extra convergence of our simulation implies a factor of three more yield, close to the factor of 3.6 to 5 observed. Matching the bang time with too small a burn region implies that the final implosion is too fast, which would lead to a narrower burn width than observed. 
EPJ Web of Conferences

\section{CONCLUSIONS}

We simulated 60 beam symmetric drive imploded capsules fired at Omega in April 2009 and September 2010 with an AMR Eulerian code. The experimental yields are 0.20 to 0.28 times the mix simulation yields, far better than the 0.05 ratio that we predict without mix. Our radius versus time plots match individual data points to within $10-25 \mu \mathrm{m}$ and the general trend is well matched. However, our simulation predicts a $40 \%$ smaller radius for the core emission radiograph. All of the available diagnostics point to our perfect capsule simulations predicting implosions that are too compressible, causing the capsule to have yields that are too high. We ran 2-D Hydra [13] simulations of capsules with the correct laser imprint pattern versus one with spherically symmetric drive and found the laser imprinting reduced the yield by a factor of 2.5. If we multiply the Eulerian mix yield ratios by this amount, we would predict ratios between 0.5 and 0.7 .

We have hypotheses on why our calculations have too high a $\mathrm{T}_{\text {ion }}$ value and too narrow a burn width. It is likely that our approximate and perfectly symmetric capsule energy source causes too perfect an implosion. The result would be too much compression and yields that are too high. It is also possible that even though we are using a mix model, not enough material is mixed. In both cases, the calculations would produce yields that are too high and core images that are too small. Preheating of the shell by hot electrons could also reduce the convergence ratio. We will conduct a detailed comparison of neutron burn history curves and use dopant layers in future shots to determine the quantity and location of mixed material to sort out which hypothesis is correct.

We thank our colleagues at Los Alamos, Rochester, and Livermore for the ongoing dialogue that offered many ideas and suggestions to improve this work. This work was performed under the auspices of the U.S. Department of Energy by Los Alamos National Security, LLC under Contract No. DE-AC52-06NA25396.

\section{References}

[1] G.R. Bennett, J.M. Wallace, T.J. Murphy, R.E. Chrien, N.D. Delamater, et al., Phys. Plasmas 7, 2594 (2000)

[2] D.D. Meyerhoffer, J.A. Delettrez, R. Epstein, V. Yu, et al., Phys. Plasmas 8, 2251 (2001)

[3] S.W. Haan, Phys Rev A 39, 5812 (1989)

[4] L. Welser-Sherrill, R.C. Mancini, D.A. Haynes, S.W. Haan, I.E. Golovkin, et al., Phys Plasmas 14, 072705 (2007)

[5] P.B. Radha, T.J.B. Collins, J.A. Delettrez, Y. Elbaz, R. Epstein, et al., Phys. Plasmas 12, 056307 (2005)

[6] B.A. Hammel, H.A. Scott, S.P. Regan, C. Cerjan, D.S. Clark, et al., Phys. Plasmas 18, 056310 (2011)

[7] N.M. Hoffman, B.A.Hammel, and O. Schilling, J. Phys, Conference Series 244, 022011 (2010)

[8] A. Banerjee, R.A. Gore, and M.J. Andrews, Phys. Rev. E 82, 046309 (2010)

[9] T.R. Boehly, D. Brown, R. Craxton, R. Keck, J. Knauer, J. Kelly, T. Kessler, S. Kumpan, S. Loucks, S. Letzring, F. Marshall, R. McCrory, S. Morse, W. Seka, J. Soures, and C. Verdon, Opt. Commun. 133, 495 (1997)

[10] G.H. Miller, E.I Moses, and C.R. Wuest, Nucl. Fusion 44, S228 (2004)

[11] E.S. Dodd, J.F. Benage, G.A. Kyrala, D.C. Wilson, F.J. Wysocki, W. Seka, V. Yu. Glebov, C. Stoeckl, and J.A. Frenje, Phys. Plasmas 19, 042703 (2012)

[12] P.A. Bradley, J.A. Cobble, I.L. Tregillis, M.J. Schmitt, K.D. Obrey, et al., Phys. Plasmas 19, 092703 (2012)

[13] M.M. Marinak, G.D. Kerbel, N.A. Gentile, O. Jones, D. Munro, S. Pollaine, T.R. Dittrich, and S.W. Haan, Phys. Plasmas 8, 2275 (2001) 